\title{
CSF1 is involved in breast cancer progression through inducing monocyte differentiation and homing
}

\author{
JINGXIAN DING ${ }^{1,2}$, CHUNGEN GUO $^{3}$, PINGHUA HU $^{3}$, JUN CHEN $^{3}$, \\ QIUMING LIU ${ }^{3}$, XIAOBO WU ${ }^{3}$, YALI CAO ${ }^{3}$ and JIONG WU $\mathrm{WU}^{2,4}$ \\ ${ }^{1}$ Department of Radiation Oncology, The Third Hospital of Nanchang, Nanchang, Jiangxi 330009; \\ ${ }^{2}$ Department of Breast Surgery, Fudan University Shanghai Cancer Center, Shanghai 200032; \\ ${ }^{3}$ Department of Breast Surgery, The Third Hospital of Nanchang, Nanchang, Jiangxi 330009; \\ ${ }^{4}$ Department of Oncology, Shanghai Medical College, Fudan University, Shanghai 200032, P.R. China
}

Received June 15, 2016; Accepted August 9, 2016

DOI: $10.3892 / \mathrm{ijo} .2016 .3680$

\begin{abstract}
Despite the great progress in breast cancer research and treatment, measures for efficient targeting of triple-negative breast cancer (TNBC) are still lacking. The well-established dependency of cancer cells on their microenvironment suggests that targeting the tumor niche might form a novel therapeutic approach. We identified the tumor-associated macrophage (TAM) infiltration in breast cancer tissues by immunohistochemistry, and analyzed overall survival (OS). U937 co-cultures with MDA-MB-231, MDA-MB-468 and MCF-7, respectively, to simulate in vivo cellular interactions were assessed. In hormone-independent breast cancer cell conditioned media (CM), U937 differentiates into M2 macrophage as identified by morphological changes and expression of specific surface antigens CD163 and CD204. Moreover, MDA-MB-231 recruits U937, and colony-stimulating factor 1 (CSF1) level in MDA-MB-231 and MDA-MB-468 CM is much higher than that of MCF-7. Overexpression of CSF1 in MCF-7 fails to rebuild its aggressiveness both in vitro and in vivo since CSF1 was not found extracellularly, while genetic inhibition of CSF1 in MDA-MB-231 abrogates TAM infiltration and consequently reduces tumorigenesis in non-obese diabetic/severe combined immunodeficient (NOD/SCID) mice. Using various strategies we demonstrate that CSF1-induced TAMs specifically support breast cancer progression. Importantly, our results may reveal the efficacy of using targeted therapy against tumor niche and indicate that CSF1 inhibition may limit some breast cancer progression.
\end{abstract}

Correspondence to: Professor Yali Cao, Department of Breast Surgery, The Third Hospital of Nanchang, South Xiangshan Road 2, Nanchang, Jiangxi 330009, P.R. China

E-mail: caoyali@medmail.com.cn

Professor Jiong Wu, Department of Breast Surgery, Fudan University Shanghai Cancer Center, Dong'An Road 270, Shanghai 200032, P.R. China

E-mail: wujiong1122@vip.sina.com

Key words: breast cancer, colony-stimulating factor 1, tumor-associated macrophage, tumor progression, cytokine

\section{Introduction}

In the most recent 5 years, delay-adjusted cancer incidence rates declined by $1.8 \%$ per year in men and were stable in women, while cancer death rates nationwide decreased by $1.8 \%$ per year in men and by $1.4 \%$ per year in women in the United States (1). However, breast cancer is still one of the most frequently diagnosed cancers in women and the leading cause of cancer-related death among women worldwide, including China, and also one of the leading causes of disease-associated death among women. Despite the great progress that has been made in breast cancer research and treatment, measures for efficient targeting triple-negative breast cancer (TNBC) are still unavailable. Colony-stimulating factor 1 (CSF1), also known as macrophage colony-stimulating factor (M-CSF) is a secreted cytokine which influences hematopoietic stem cells to differentiate into macrophages or other related cell types, and is involved in the proliferation, differentiation and survival of monocytes, macrophages and bone marrow progenitor cells $(2,3)$. The active form of CSF1 is found extracellularly as a disulfide-linked homodimer, and has been reported to be correlated with poor prognosis in many cancers (4). It is reported that about one in eight women in the United States will develop invasive breast cancer over the course of their lifetime, and TNBC is one subtype with the poorest prognosis, which accounts of $15-25 \%$ breast cancers (1). Metastasis is a far from known, complex, multi-step process, including at least these six steps: tumor cell invasion of the basement membrane, intravasation into the vascular or lymphatic system, survival in the blood circulation or lymph nodes, attachment to the blood vessel wall and extravasation to the target organ, followed by subsequent colonization and aggressive growth to form a macrometastasis under a favorable microenvironment (5). Each step makes cancer metastasis an almost impossible mission. However, in clinical practice, a number of subjects with early-stage breast cancer even die from relapse or metastasis, regardless of the intensive adjuvant therapy given. Substantial efforts have been made in understanding the molecular mechanisms of the disease and applying adjuvant therapy to decrease the probabilities of relapse and metastasis, such as targeted therapy and endocrine therapy; however, there 
is no such an effective measure to patients with TNBC. The role of tumor microenvironment (TME) during the initiation and progression of breast cancer is now realized to be of great importance, both for the understanding of breast cancer biology and exploiting new molecular targets for breast cancers. Macrophage is a major component of TME, and its infiltration is not an uncommon phenomenon in cancer tissues. Normally, these macrophages are called tumor-associated macrophages (TAMs), which share properties of alternatively activated macrophage (M2) phenotype (6). Reports indicate that the prevalence of TAM infiltration correlates with poor prognosis in some cancer patients indicating a macrophage-supporting role for tumor progression $(7,8)$. However, the exact mechanisms involved are still ambiguous.

Macrophage originates from the mononuclear phagocytic lineage, whose polarization is dependent on the cytokines in the microenvironment. The classical activation (M1) is triggered by $\mathrm{T}$ helper 1 (Th1) cytokines, such as interferon- $\gamma$, bacterial lipopolysaccharide (LPS) and TNF- $\alpha$. In contrast, the alternative activation (M2) is induced by T helper 2 (Th2) cytokines, like IL-4, IL-13 and CSF1 (M-CSF) (9,10). Classical or M1 activation mediates the defense against bacterial pathogens, and secretes high levels of IL-12 and low levels of IL-10, whilst alternative or M2 activation has a 'pro-tumorigenic' effect by producing high levels of IL-10, TGF- $\beta$ and low levels of IL-12. TAMs present the M2 phenotype in TME, and seem to actively promote tumor growth $(11,12)$. TAMs differentiate from circulating monocytes that leave the vasculature and enter the tumor tissue in response to a variety of cues secreted from the tumor and also in response to TME. Once there, they have been shown to promote angiogenesis, tumor growth, invasion and metastasis through secretion of cytokines to coordinate tumor-promoting immune responses as well as through secretion of tissue-remodeling cysteine cathepsin proteases (13).

In this study, the objective was to examine whether CSF1 level and TAM infiltration correlates with breast cancer hormone receptor status, which influences treatment measures and cancer prognosis. Further, to clarify the mechanism involved may usher in an era of new treatment for CSF1 expression breast cancer, especially for those hormone-independent TNBCs with no well-established targeted therapy available.

\section{Materials and methods}

Ethics statement. The study involving human participants was approved by the Ethics Committee of Fudan University Shanghai Cancer Center. Written informed consent was obtained from all the patients before the enrollment. All animal protocols were approved by the Animal Care Committee of Shanghai Institutes for Biological Sciences, Chinese Academy of Sciences (permit no. IBS14-0806). Animals were kept under stable temperature and humidity conditions. All surgery was performed by cervical dislocation to minimize suffering.

Immunohistochemistry. Paraffin-embedded blocks were obtained from the Breast Malignancy Database established by the Department of Breast Surgery, Fudan University Shanghai Cancer Center (Shanghai, China). All of the enrolled patients have fully detailed clinicopathological information and follow-up results. Written informed consent was obtained from the patients before enrollment. We are authorized to use the tissues for research only and have reported the database information previously (14). For immunohistochemical analysis, 89 paraffin-embedded blocks were cut into $5-\mu \mathrm{m}$ serial sections, and following the confirmation of breast cancer diagnosis by H\&E staining, immunohistochemistry was performed following standard procedures. CD163 antibody (clone 10D6; Novocastra/Leica Microsystems, Newcastle, UK) used for immunostaining was titered to find the optimal concentration (1:100). Sections were counterstained with hematoxylin for the identification of the nuclei. Detection was performed using the Dako EnVision System. Images were captured under a microscope with a CCD camera.

Cell culture. Breast cancer cell lines MDA-MB-231, MD-MB-468 and MCF-7 were originally obtained from the American Type Culture Collection (ATCC; Manassas, VA, USA); the human promonocytic cell line U937 originating from the ATCC was kindly provided by Professor Duan Ma (Key Laboratory of Molecular Medicine, Ministry of Education, Shanghai Medical College, Fudan University). All cells were maintained in RPMI-1640 supplemented with $10 \%$ fetal bovine serum (FBS) and $100 \mathrm{U} / \mathrm{ml}$ penicillin/streptomycin (Gibco/Invitrogen, Carlsbad, CA, USA) at $37^{\circ} \mathrm{C}$ in a humidified atmosphere of $5 \% \mathrm{CO}_{2}$.

Lentivirus infected stable cell line generation. MDA-MB-231, MD-MB-468, MCF-7 and U937 cells were transfected with green or red fluorescence protein (GFP or RFP) expressing lentiviral particles (cat. nos. LVP340 and LVP299; GenTarget, Inc., San Diego, CA, USA) according to the manufacturer's instructions. MCF-7 was transfected with GFP with puromycin resistance (GFP-puro)-tagged CSF1-overexpressing lentiviral particles (cat. no. GCK970139; GeneChem Co., Ltd., Shanghai, China), and MDA-MB-231 was transfected with GFP-puro-tagged shRNA lentiviral particles specifically targeting CSF1 (cat. no. ST3071-A; Shanghai SunBio Medical Biotechnology Co., Ltd., Shanghai, China). Briefly, for adhesive cells the cells were seeded in complete medium at appropriate density (at the time of transduction, cells should be $50-75 \%$ confluent) and incubated overnight, the medium was removed and fresh warm complete medium was added with appropriate amount of pre-made lentivirus to obtain the desired MOI, then incubated for another $72 \mathrm{~h}$ at $37^{\circ} \mathrm{C}$ in a humidified atmosphere of $5 \% \mathrm{CO}_{2}$. For suspension cells, the cells were seeded in complete medium at appropriate density and incubated until density reaching $3 \times 10^{6}$ cells $/ \mathrm{ml}$, then cells were diluted into $1 \times 10^{6}$ cells/ml with fresh warm complete medium and appropriate amount of pre-made lentivirus was added to obtain the desired MOI, incubated for $24 \mathrm{~h}$ at $37^{\circ} \mathrm{C}$ in a humidified atmosphere of $5 \% \mathrm{CO}_{2}$ with a shaking flask, then an equal amount of fresh medium containing relevant antibiotics was added for another $48 \mathrm{~h}$. Positive transduction of cells were visualized by fluorescence microscopy and isolated by fluorescence-activated cell sorting (FACS) followed by puromycin or neomycin selection for 2 weeks.

Transwell assay with or without extracellular matrix barrier. For the co-culture assay, MDA-MB-231, MDA-MB-468, 
MCF-7 and U937 cells cultured in RPMI-1640 supplemented with $10 \% \mathrm{FBS}$ and $100 \mathrm{U} / \mathrm{ml}$ penicillin/streptomycin at a final concentration of $5 \times 10^{5} / \mathrm{ml}$ using 6-well insert system with $0.4-\mu \mathrm{m}$ pores (cat. no. 3450 ; Corning, Inc., Corning, NY, USA). Breast cancer cell suspension $(1 \mathrm{ml})$ was added to each insert well and $1 \mathrm{ml}$ of U937 cell suspension was added to each lower well. After a 72-h incubation, U937 cells in the lower well were harvested for further examinations. Microscopy images were captured for each well at five randomly picked fields under a microscope with a 10X objective lens, and the number of adhesive cells for each field was quantified. The number of adhesive cells was determined by comparing different breast cancer cell lines and blank control, which was used as reference.

For the Transwell migration assay, GFP expression MDA-MB-231, MCF-7 stable cells were cultured in complete RPMI-1640 medium and RFP expression U937 stable cells cultured in serum-free RPMI-1640 using a 24-well insert system with $5-\mu \mathrm{m}$ pores (cat. no. 3421 ; Corning, Inc.). A total of $0.5 \mathrm{ml}$ of $5 \times 10^{5} / \mathrm{ml}$ breast cancer cell suspension was added to each lower well, and after a 24-h incubation, $0.5 \mathrm{ml}$ of $5 \times 10^{5} / \mathrm{ml}$ U937 cell suspension was added to each insert well. The breast cancer conditioned medium in the lower chamber served as a chemoattractant. After a 72-h incubation, migratory cells were visualized by fluorescence microscopy and images were captured under a fluorescence microscope with a CCD camera. Microscopy images were captured for each well at five randomly picked fields with a 10X objective lens, and the number of migratory cells for each field was quantified. The number of migratory cells was determined by comparing different breast cancer cell lines and blank control, which was used as reference.

The invasion assay was performed similarly to migration assay using $24-w e l l$ insert system with $8-\mu \mathrm{m}$ pores (cat. no. 3422; Corning, Inc.) except the inside of the insert was precoated with a thin layer of Matrige ${ }^{\mathrm{TM}}$ Basement Membrane Matrix (BD Biosciences) diluted with serum-free medium to a final concentration of $6 \mathrm{mg} / \mathrm{ml}$. A total of $0.5 \mathrm{ml}$ of $5 \times 10^{5} / \mathrm{ml}$ breast cancer cell suspension was added to each lower well, and after a $24-\mathrm{h}$ incubation, $0.5 \mathrm{ml}$ of $5 \times 10^{5} / \mathrm{ml}$ U937 cell suspension was added to each insert well. The breast cancer conditioned medium in the lower chamber served as a chemoattractant. After a 72-h incubation, invaded cells were visualized by fluorescence microscopy and images were captured under a fluorescence microscope with a CCD camera. Microscopy images were captured for each well at five randomly picked fields with a 10X objective lens, and the number of invaded cells for each field was quantified. The number of invaded cells was determined by comparing different breast cancer cell lines and blank control, which was used as reference.

Measurement of cytokine production. Cytokine secretion was quantified by Bio-Plex ${ }^{\circledR}$ cytokine assay. Briefly, conditioned medium samples were obtained after centrifugation to remove cells and their debris and stored at $-80^{\circ} \mathrm{C}$ until cytokine profiling. We used the fluorescent bead-based detection assay system, with an array of beads in liquid suspension, each containing different ratios of two spectrally distinct fluorophores, thereby assigning a unique spectral identity. The beads, which had been conjugated with a monoclonal antibody specific for a target protein, were incubated with the samples to be tested, washed, followed by addition of a biotinylated detection antibody, washed again, and finally incubated with streptavidin-PE. A wide range of standards $(1.78-29,107.00 \mathrm{pg} / \mathrm{ml})$ was used to enable quantitation of the individual cytokines using a Bio-Plex array reader with a dual laser detector and real-time digital signal processing. Cytokine levels were measured using a Multiplex kit (Bio-Rad Laboratories, Inc., San Diego, CA, USA) according to the manufacturer's instructions. Standard curves for each cytokine were generated using the reference concentrations provided in the kit. The plate was run on a Luminex 200 Bio-Plex Instrument (Bio-Rad, Hercules, CA, USA). Raw fluorescence data were analyzed with software using the 5-parameter logistic method. Detailed procedures of the Bio-Plex ${ }^{\circledR}$ Suspension Array System have been described elsewhere (15-17).

Reverse transcription-PCR analysis. Total RNA was isolated from $1 \times 10^{6}$ cells using TRIzol (Invitrogen) according to the manufacturer's instructions. One microgram of total RNA was reverse transcribed using PrimeScript ${ }^{\circledR}$ RT-PCR Kit (Takara Bio, Dalian, China) primed with oligo(dT). One microliter of cDNA was subjected to PCR amplification using gene-specific primers. PCR products were resolved on a $2 \%$ agarose gel and visualized by ethidium bromide staining. Quantitative PCR was carried out using the $\mathrm{SYBR}^{\circledR}$-Green PCR Master Mix Kit (Takara Bio). Primer sequences are detailed in Table I. The quantification of gene expression was normalized to the expression of GAPDH.

Western blotting assay. For immunoblot analysis, cell lysates containing $50 \mu \mathrm{g}$ of total protein were separated by electrophoresis on $10 \%$ sodium dodecyl sulfate-polyacrylamide gels and electroblotted onto polyvinylidene difluoride membranes (EMD Millipore, Billerica, MA, USA). After blocking of the unoccupied sites with 5\% skim milk-PBS, the membranes were probed with the desired primary antibodies at $4^{\circ} \mathrm{C}$ overnight, followed by incubation with the appropriate HRP-conjugated secondary antibodies, according to the manufacturer's instructions. The primary antibodies were anti- $\beta$-actin, anti-CSF1, and the proper dilution of them was according to the instructions. The signal was visualized by ECL Western Blotting Detection Reagents (Pierce Biotechnology, Inc.) and photographed with ImageQuant LAS 4000 (GE Healthcare, Piscataway, NJ, USA). $\beta$-actin was used as a loading control.

Breast cancer xenografts. To assess the tumorigenicity in vivo, 8 -week-old female non-obese diabetic/severe combined immunodeficient (NOD/SCID) mice were used. Eighteen mice were assigned to six groups equally. A total of $5 \times 10^{5}$ cells suspended in $100 \mu \mathrm{l}$ PBS were injected orthotopically into the right lower mammary fat pad according to standard injection procedures. Once tumors were palpable, tumor volume $\left(1 / 2 \times\right.$ length $\mathrm{x}$ width $\left.{ }^{2}\right)$ was monitored every other day for 8 weeks. Tumor growth curves were plotted.

Statistical analysis. The quantitative results are presented as the mean \pm standard error of at least three independent experiments. Significant differences were determined with 

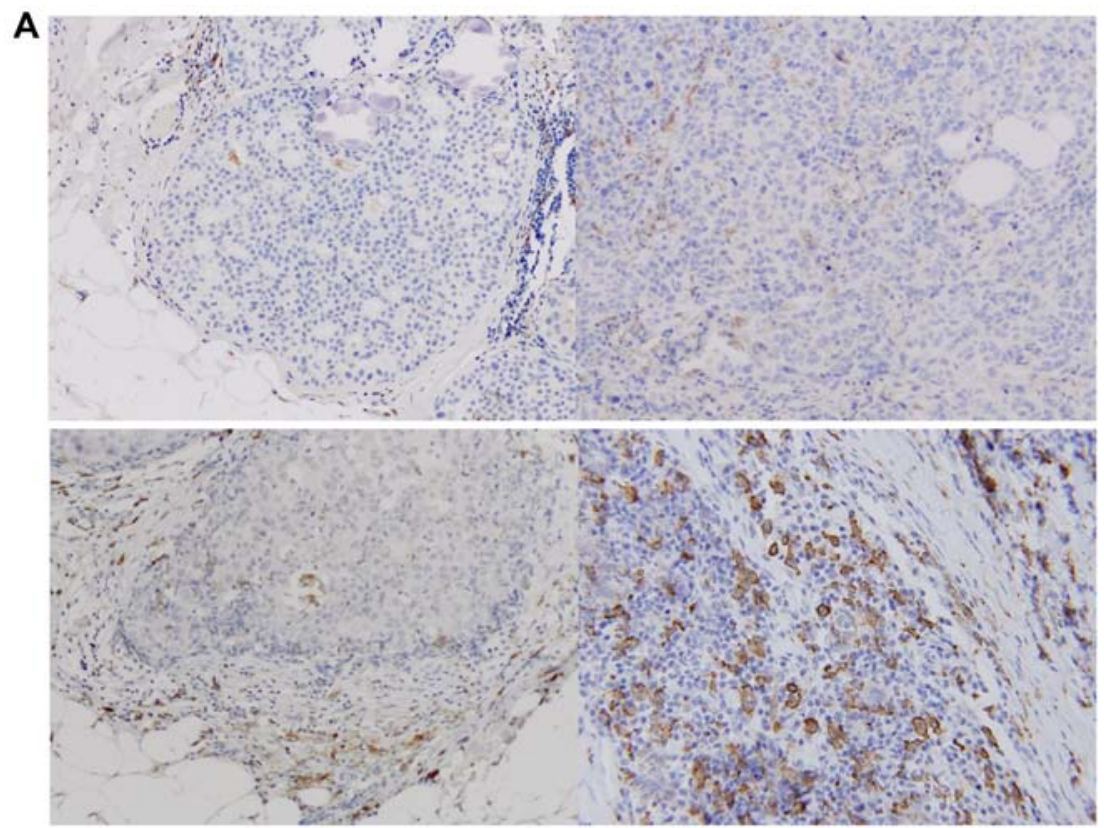

B

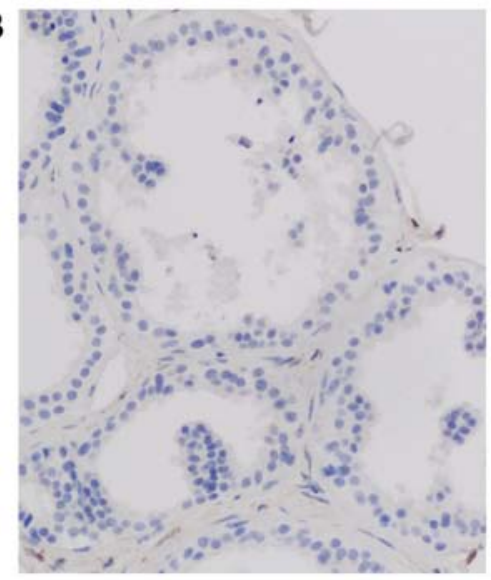

C

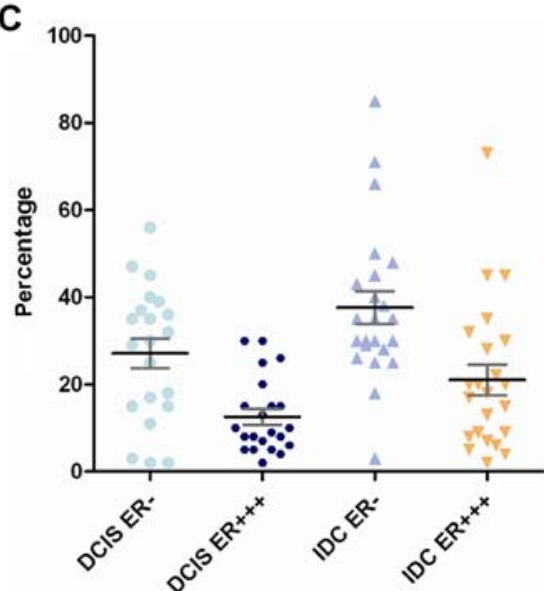

Figure 1. Macrophage infiltration in primary breast cancer. IHC staining of formalin-fixed breast cancer samples for CD163. (A) Representative images showing CD163 expression in ER + DCIS, ER + IDC, ER-DCIS, ER-IDC, respectively. (B) CD163 expression in normal breast tissues as control. (C) Expression levels were compared in breast cancer according to ER status, showing high $\mathrm{CD}_{163^{+}}$macrophage infiltration in ER breast cancer $(\mathrm{p}=0.004)$. Bars represent the mean \pm SD.

Student's t-test using Excel or GraphPad Prism 5 Demo software (GraphPad Software, Inc., San Diego, CA, USA). $\mathrm{P}<0.05$ was considered statistically significant.

\section{Results}

The prevalence of TAM infiltration is significantly higher in hormone-independent breast cancer samples compared with hormone-dependent counterparts. TAMs play an important role in the growth and progression of cancers. CD163 has been reported as a specific surface antigen of M2 macrophages (12). Herein, we first detected CD163 expression in breast cancer tissues or non-cancer breast tissues by immunohistochemistry. The results showed that the percentage of CD163-positive cells was significantly higher in hormone-independent breast cancers than that in hormone-dependent counterparts and normal breast tissues, in some cases, TAMs may make up as much as 50\% of tumor volume. Moreover, the difference was also significant between hormone-independent DCIS and IDC, whereas CD163
Table I. Characteristics of PCR primer sets and products.

\begin{tabular}{lcl}
\hline Gene & $\begin{array}{c}\text { Size of PCR } \\
\text { product }(\mathrm{bp})\end{array}$ & \multicolumn{1}{c}{$\begin{array}{c}\text { Primer sequences } \\
\left(5^{\prime} \rightarrow 3^{\prime}\right)\end{array}$} \\
\hline GAPDH & 200 & $\begin{array}{l}\text { F acccagaagactgtggatgg } \\
\text { R tctagacggcaggtcaggtc }\end{array}$ \\
CD163 & 146 & $\begin{array}{l}\text { F cgagttaacgccagtaagg } \\
\text { R gaacatgtcacgccagc }\end{array}$ \\
CD204 & 366 & $\begin{array}{l}\text { F ccagggacatgggaatgcaa } \\
\text { R ccagtgggacctcgatctcc } \\
\text { F tagccacatgattgggagtgga } \\
\text { R ctcaatgtaattggcacgaggtc }\end{array}$ \\
CSF1 & 88 &
\end{tabular}

F, forward; R, reverse; CSF1, colony-stimulating factor 1 .

expression was hardly detectable in normal breast tissues (Fig. 1). However, we did not observe a significant difference among 

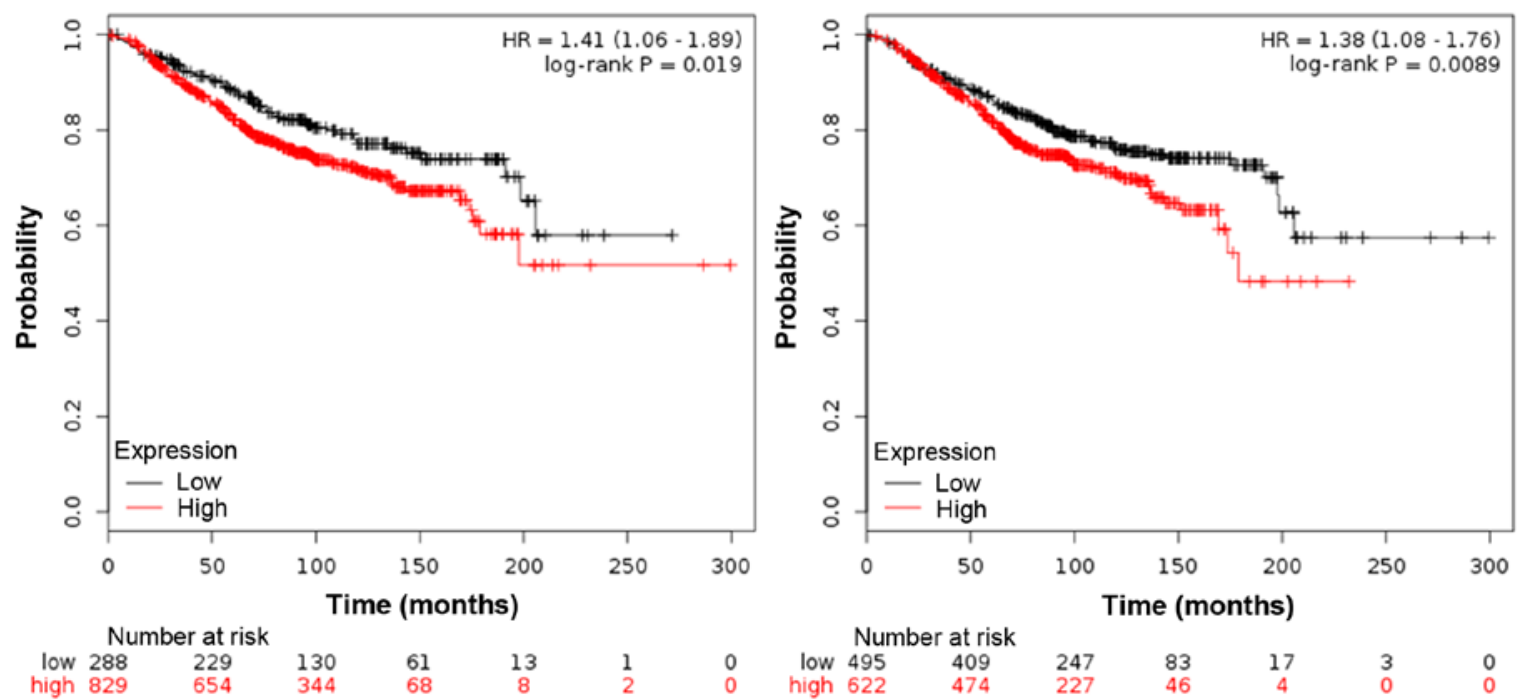

Figure 2. The correlation of TAM infiltration with OS. Kaplan-Meier survival analysis of expression of M2 macrophage-specific markers CD163 and CD204 grouped into high and low expression. High macrophage infiltration predicts decreased OS in patients with breast cancer. TAM, tumor-associated macrophage; OS, overall survival.

tumor size, HER2 expression, lymph node involvement, proliferation index (Ki67) and menstrual status (Table II).

Impact of TAM infiltration on breast cancer survival. The impact of TAM infiltration on overall survival (OS) was evaluated in breast cancer patients. Both CD163 and CD204 are specific surface antigens of M2 macrophages, as previously reported $(18,19)$. We assessed CD163 and CD204 mRNA expression level in a cohort of 1,117 breast cancer tissue samples (containing tumor stroma) based on publically available gene expression datasets (20). For $\mathrm{CD} 63^{+}$macrophage frequency, patients were grouped as 'high' or 'low' using the lower quartile as a cut-off point; while for $\mathrm{CD} 204^{+}$macrophage frequency, patients were grouped as 'high' or 'low' using the median as a cut-off point. Increased expression of CD163 and CD204 was observed in ER-negative patients, and the patients with high density of $\mathrm{CD} 163^{+}$and $\mathrm{CD} 204^{+}$macrophages also showed a poor OS, which was an independent poor prognostic factor for OS (Fig. 2).

Hormone-independent breast cancer cells influence monocyte polarization toward TAMs. The human promonocytic U937 cell line was used as a model to simulate the interaction between monocytes and breast cancer cells in vitro. U937 cells normally grow in suspension and have a smooth surface, as previously reported (21). However, after co-cultured with hormone-independent breast cancer cell lines MDA-MB-231 and MDA-MB-468 in a 6-well insert system with $0.4-\mu \mathrm{m}$ pores, they differentiated into M2 macrophages, as evidenced by a thorny morphology, large cell volume and increase in adherence, and increasing expression of surface antigen CD163 and CD204, all of which are characteristics of M2 macrophages. Whereas, no further obvious changes were observed when co-cultured with hormone-dependent MCF-7 cell line (Fig. 3).

MDA-MB-231 cancer cells recruit monocyte homing in vitro. To determine the factors that contributed to monocyte differentiation leaving the vasculature and entering the tumor tissue,
Table II. TAM infiltration in relation to clinicopathological characteristics of the breast cancer patients.

\begin{tabular}{lccc}
\hline & \multicolumn{2}{c}{ CD163 ${ }^{+}$macrophages } & \\
\cline { 2 - 3 } & Low n (\%) & High n (\%) & P-value \\
\hline $\begin{array}{l}\text { No. of patients } \\
\text { Tumor size (cm) }\end{array}$ & $22(24.7)$ & $67(75.3)$ & \\
$\leq 2$ & & & \\
$>2$ & $12(27.3)$ & $32(72.7)$ & 0.667 \\
ER status & $10(23.3)$ & $33(76.7)$ & \\
+ & & & \\
- & $18(39.1)$ & $27(60.9)$ & 0.001 \\
HER2 overexpression & $4(9.1)$ & $40(90.9)$ & \\
$\quad$ Yes & $3(20.0)$ & $12(80.0)$ & 0.642 \\
$\quad$ No & $19(25.7)$ & $55(74.3)$ & \\
$\begin{array}{l}\text { Lymph node } \\
\text { involvement }\end{array}$ & & & \\
$\quad$ Yes & $3(21.4)$ & $11(78.6)$ & 0.756 \\
$\quad$ No & $19(25.3)$ & $56(74.7)$ & \\
$\begin{array}{l}\text { Proliferation index } \\
\text { (Ki67) (\%) }\end{array}$ & & & \\
$>15$ & $18(24.3)$ & $56(75.7)$ & 0.848 \\
$<15$ & $4(26.7)$ & $11(73.3)$ & \\
Menstrual status & & & \\
Premenopausal & $9(20.5)$ & $35(79.5)$ & 0.356 \\
Postmenopausal & $13(28.9)$ & $32(71.1)$ & \\
\hline
\end{tabular}

$\mathrm{CD}_{163^{+}}$macrophage count was divided into low and high by the lower quartile value as a cut-off point based on our IHC results. High level of TAM infiltration was associated with ER-negative status. The cut-off value for Ki67 is based on breast cancer intrinsic subtype luminal A or B, whose treatment strategies and prognosis may be completely different in clinical setting. TAM, tumor-associated macrophage. 

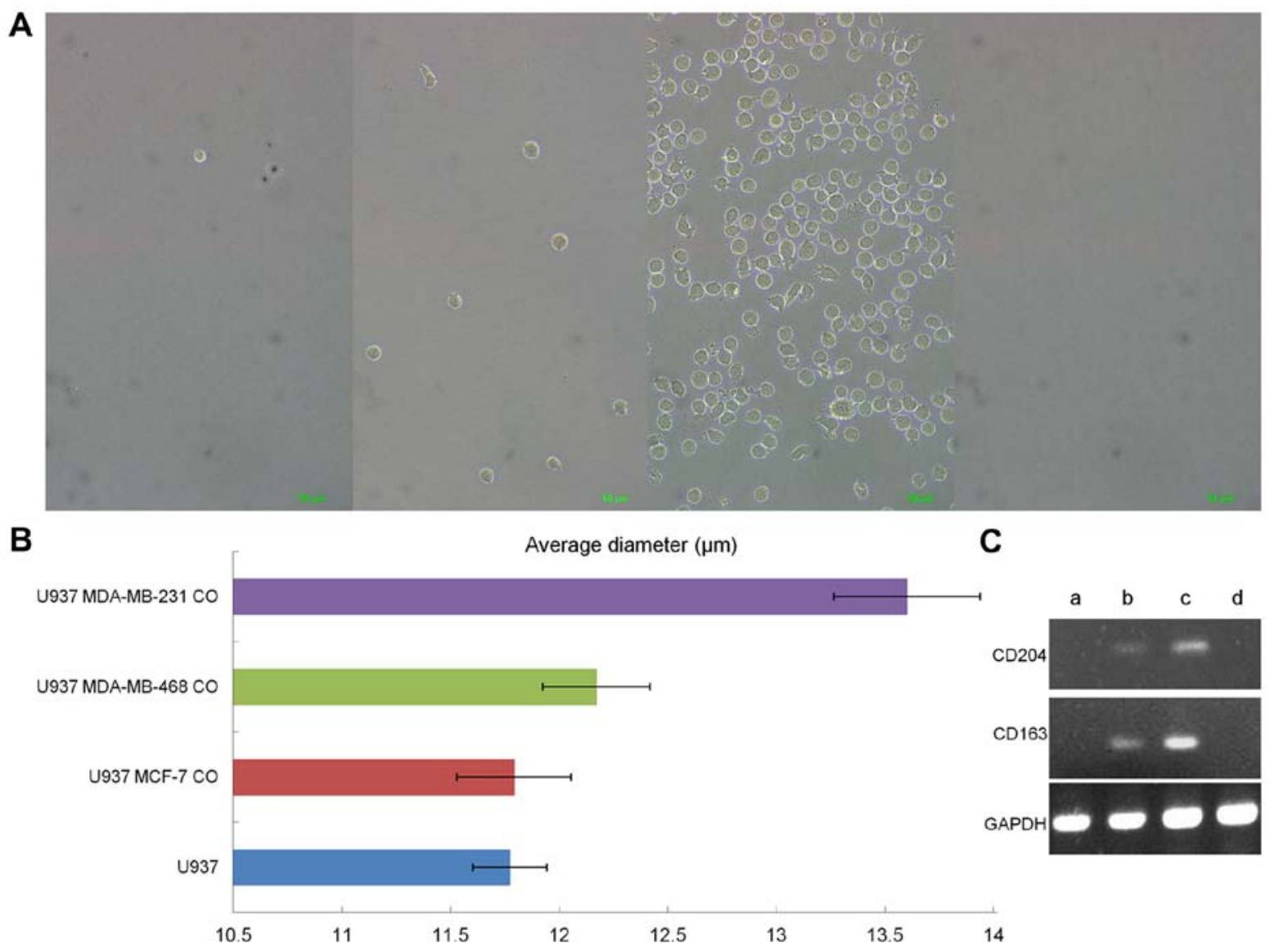

Figure 3. Differentiation of U937 monocytes into macrophages in breast cancer cell conditioned medium. U937 monocytes were co-cultured for 3 days with breast cancer cell lines to induce macrophage differentiation. (A) Bright-field imaging shows a thorny morphology and increased adherence after co-culture with MCF-7, MDA-MB-468, MDA-MB-231 or alone. (B) Cell diameter measured by a cell analyzer. (C) Increasing expression of M2 macrophage-specific surface antigen CD163 and CD204. The gels have been run under the same experimental conditions following the protocol, and GAPDH is the inner control. Lanes a-d, co-culture with MCF-7, MDA-MB-468, MDA-MB-231 or alone.

we generated stably expressing GFP-puro MDA-MB-231, MCF-7 and RFP with neomycin resistance (RFP-neo) U937 cell lines using lentiviral particles according to the protocol.

We performed the Transwell assay without extracellular matrix barrier to study the capability of breast cancer cells to recruit monocytes using a 24 -well insert system with $5-\mu \mathrm{m}$ pores. After co-cultured for $72 \mathrm{~h}$, migratory cells were visualized by fluorescence microscopy and images were captured under a fluorescence microscope. Microscopy images were captured for each well at five randomly picked fields with a 10X objective lens, and the number of migratory cells for each field was quantified (Fig. 4). We further mimicked the in vivo circumstance applying the Transwell assay with extracellular matrix barrier (BD Biosciences) using a 24-well insert system with $8-\mu \mathrm{m}$ pores. Our results showed that co-cultured MDA-MB-231 with U937 caused an increase in migration and invasion compared with $\mathrm{MCF}-7$, which indicated that hormone-independent breast cancer cells recruited more monocytes than hormone-dependent breast cancer cells (Fig. 5).

Hormone-independent breast cancer cells secrete larger amount of monocyte differentiation-related cytokine CSF1. The fluorescent bead-based detection assay is a highly sensitive method for profiling multiple cytokines, which has an advantage over conventional enzyme-linked immunosorbent assay (ELISA), which enables analysis of a number of analytes simultaneously (22). The strong effects of the MDA-MB-231 cells in monocyte recruitment and differentiation led us to inspect their conditioned media (CM) for dissimilarly secreted monocyte chemotaxis and differentiating factors. Among the cytokines tested, there was a significant difference in CSF1 secretion level among different breast cancer cell lines. We found that mean CSF1 level was much higher in MDA-MB-231 cell conditioned medium compared to that of MCF-7 (Fig. 6).

Overexpression of CSF1 in MCF-7 fails to rebuild its aggressiveness both in vitro and in vivo while genetic inhibition of CSF1 abrogates TAM infiltration and consequently reduces tumorigenesis in $M D A-M B-231$. In order to further study the correlation of CSF1 secretion level with monocyte differentiation and homing, and with tumorigenic ability, we overexpressed CSF1 in MCF-7 cells and generated the stable cell line using lentivirus particles following the protocol. The CSF1-overexpressing MCF-7 stable cells were identified by RT-PCR and western blotting, which showed that CSF1 was overexpressed in MCF-7 artificially (Fig. 7A and B). However, CSF1 level was not increased in MCF-7 CM (Fig. 7C).

We co-cultured CSF1-overexpressing MCF-7 stable cells with U937 cells to identify its ability to induce monocyte differentiation using Transwell assay in vitro. We observed 


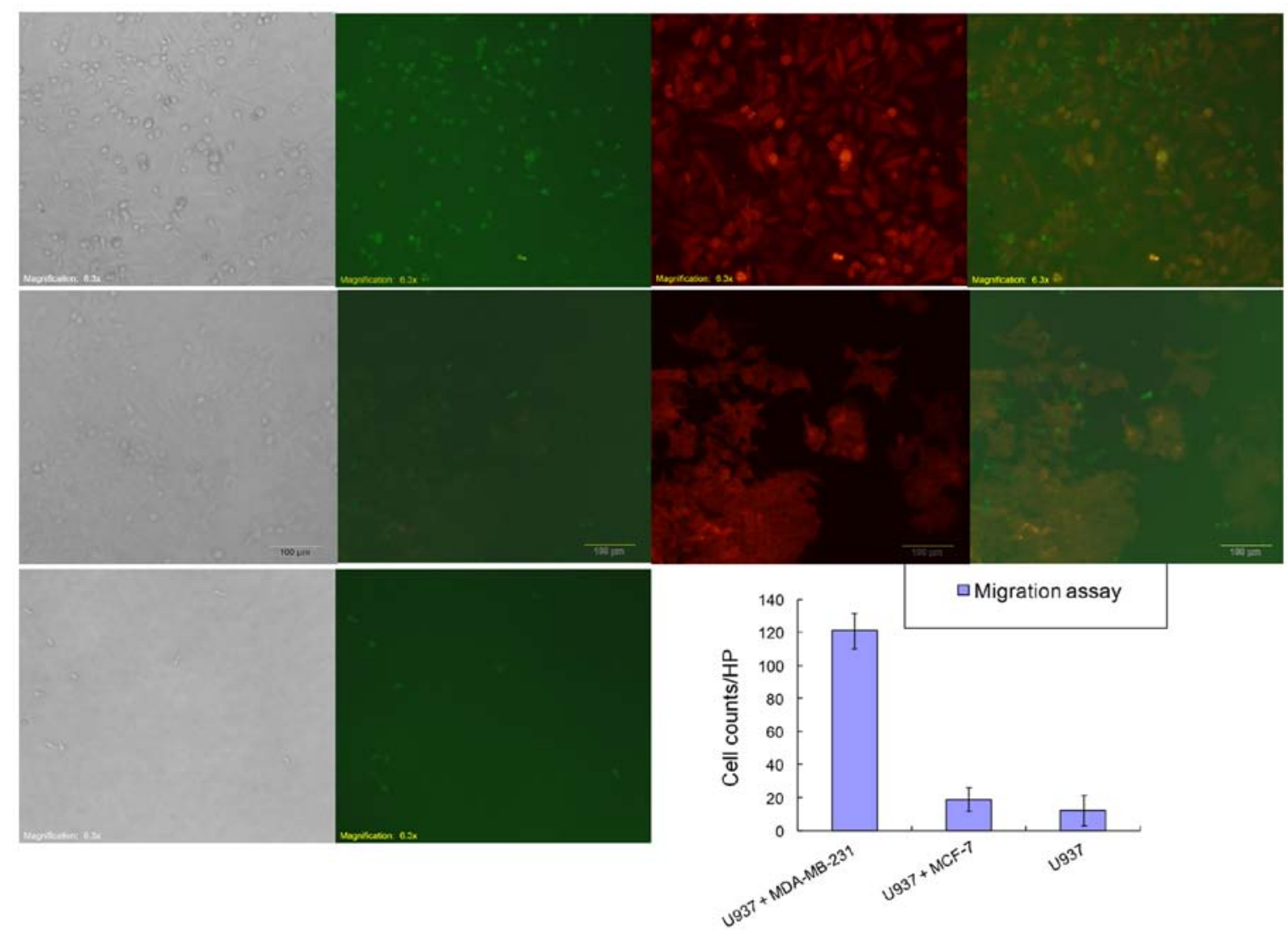

Figure 4. Effects of breast cancer cells on U937 cell migration. U937 monocytes were co-cultured for 3 days with breast cancer cell lines to study the effects of breast cancer cell conditioned medium on cell migration. Upper, U937 + MDA-MB-231; middle, U937 + MCF; lower, U937 alone. The number of migratory U937 cells was much higher in the presence of MDA-MB-231 than MCF-7 cells. Bars represent the mean \pm SD. All experiments were repeated three times. P-values were generated using a two-sided t-test.

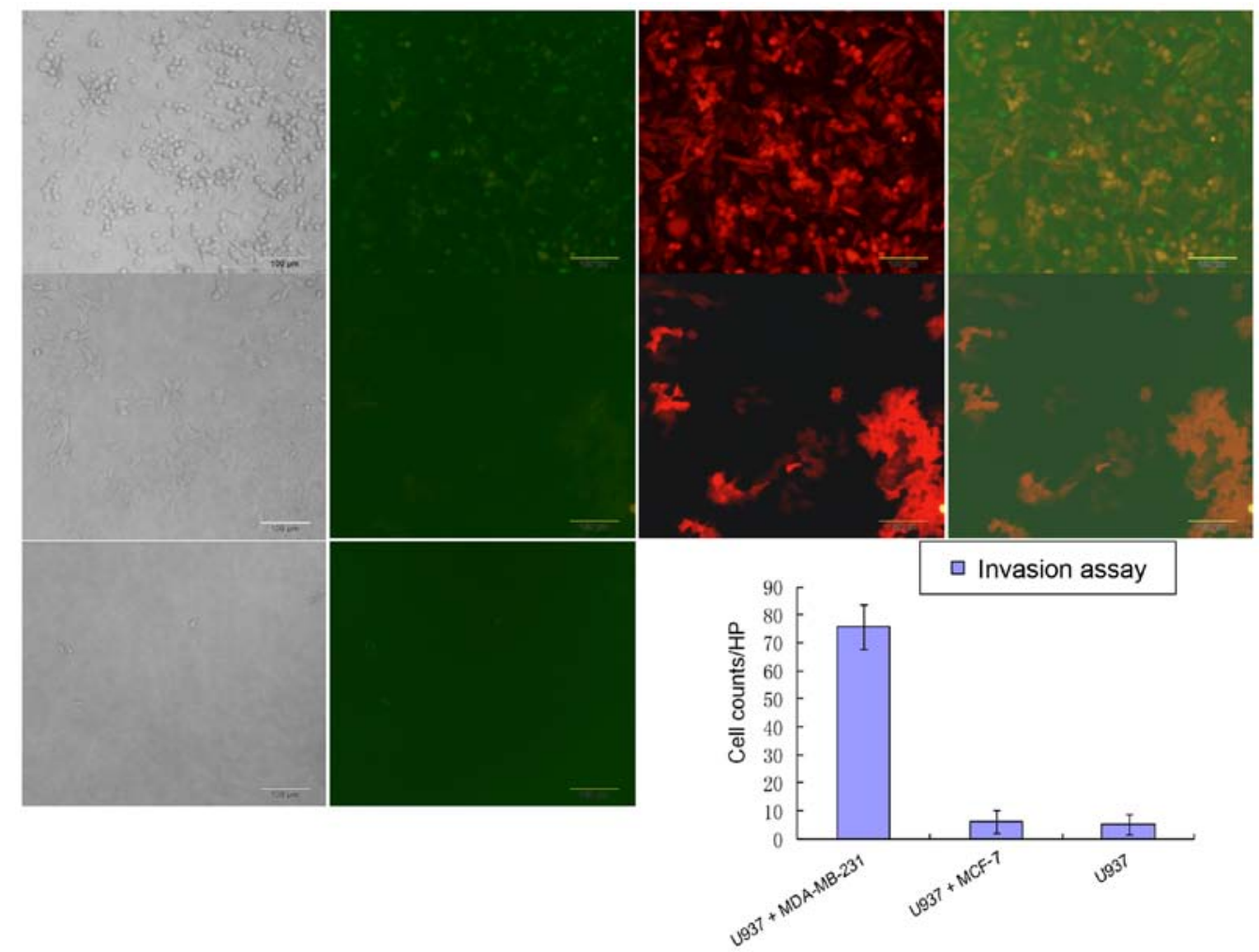

Figure 5. Effects of breast cancer cells on U937 cells homing in vitro. Invasive ability of U937 cells was assessed by Transwell inserts with Matrigel Upper, U937 + MDA-MB-231; middle, U937 + MCF; lower, U937 alone. The number of invaded U937 cells was much higher in the presence of MDA-MB-231 than MCF-7 cells. Bars represent the mean \pm SD. All experiments were repeated three times. P-values were generated using a two-sided t-test. 

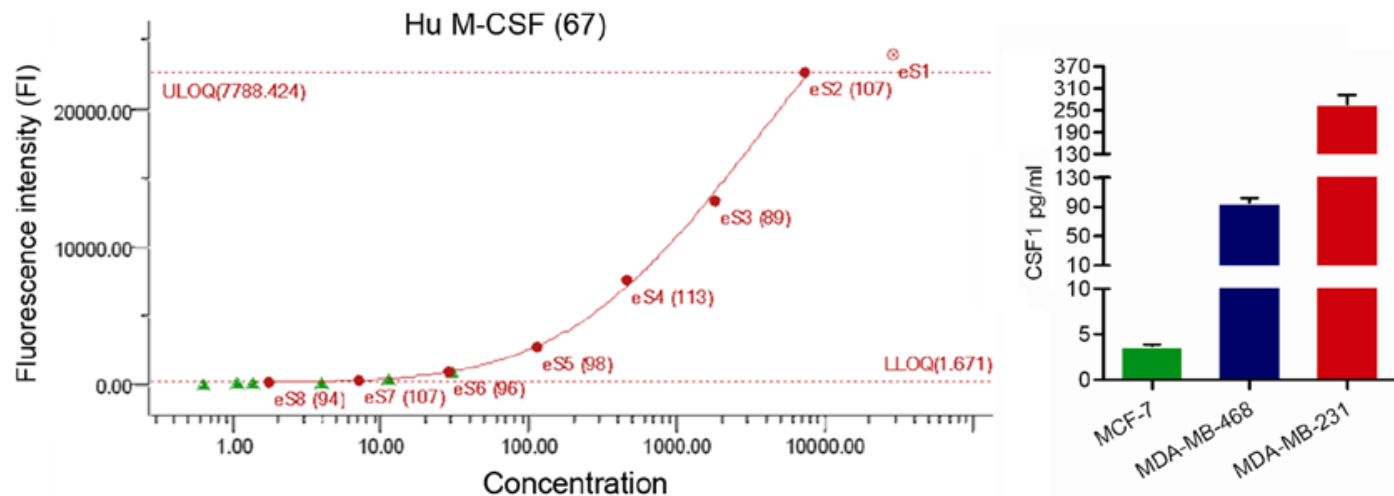

Figure 6. CSF1 secretion levels among MCF-7, MDA-MB-468 and MDA-MB-231 conditioned medium. Mean CSF1 level was much higher in MDA-MB-231 and MDA-MB-468 conditioned medium compared to that of MCF-7, $\mathrm{p}=0.001$. The upper is the standard curve of the CSF1 (M-CSF) inner control. CSF1, colony-stimulating factor 1 ; M-CSF, macrophage colony-stimulating factor.
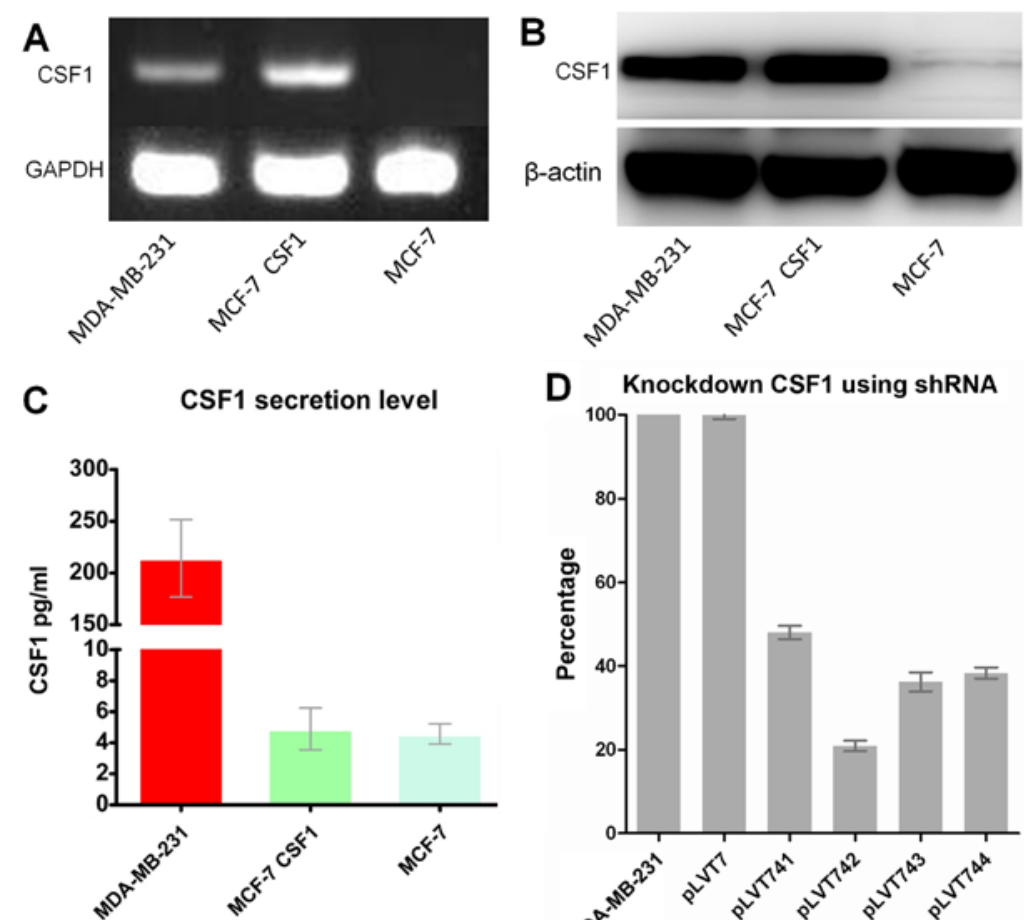

D Knockdown CSF1 using shRNA

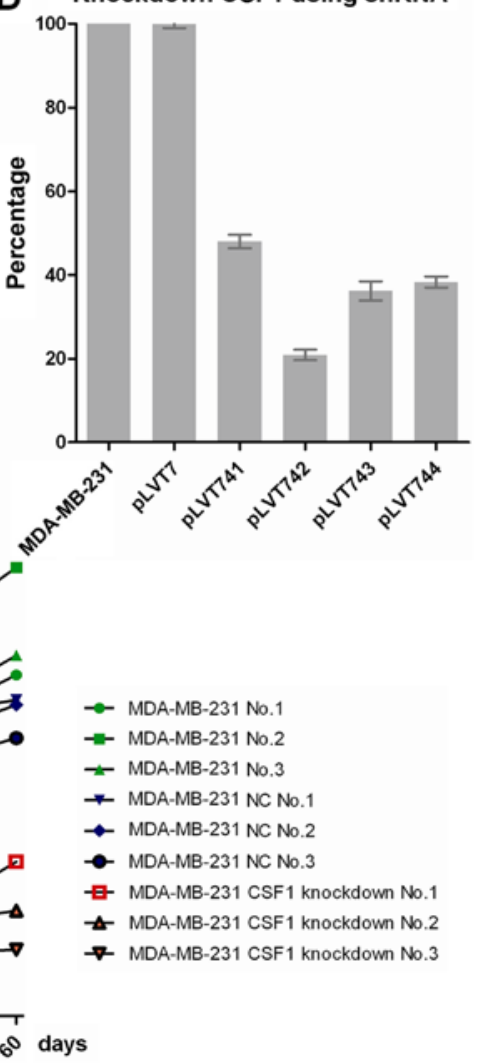

Figure 7. The effects of CSF1 on the biological behavior of breast cancer cell lines. Overexpression of CSF1 in MCF-7 fails to rebuild its aggressiveness both in vitro and in vivo since CSF1 not found extracellularly, while genetic inhibition of CSF1 abrogates TAM infiltration and consequently reduces tumorigenesis in MDA-MB-231. (A and B) Successful overexpression of CSF1 in MCF-7 was identified by RT-PCR and western blotting, respectively. The gels have been run under the same experimental conditions following the protocol, and GAPDH and $\beta$-actin are the inner controls. (C) No obvious increase of CSF1 level in CSF1-overexpressing MCF-7 cell conditioned medium compared with MCF-7 cells. MDA-MB-231 was applied as a positive control. (D) CSF1 expression after shRNA lentivirus infection by real-time PCR. (E) Only high CSF1 secretion level cell line MDA-MB-231 formed a xenograft tumor in female NOD/SCID mice during the first 6 weeks. Tumor growth curves were drawn individually until 8 weeks after injection. No xenograft tumors were observed in MCF-7, MCF-7 NC or MCF-7 CSF1 overexpression group. CSF1, colony-stimulating factor 1; TAM, tumor-associated macrophage; NOD/SCID, non-obese diabetic/severe combined immunodeficient. 
Table III. Information on shRNA and target sequences.

\begin{tabular}{lcllr}
\hline Marker & Gene & Gene ID & TargetSeq & GC $(\%)$ \\
\hline pLVT741 & CSF1 & NM_000757 & CCTCGTGCCAAATTACATT & 42.1 \\
pLVT742 & CSF1 & NM_000757 & CCATGCGCTTCAGAGATAA & 47.4 \\
pLVT743 & CSF1 & NM_000757 & GCCAAGATGTGGTGACCAA & 52.6 \\
pLVT744 & CSF1 & NM_000757 & GGATGACAGACAGGTGGAA & 52.6 \\
pLVT7 & NC & & TTCTCCGAACGTGTCACGT & 52.6 \\
\hline
\end{tabular}

CSF1, colony-stimulating factor 1 .

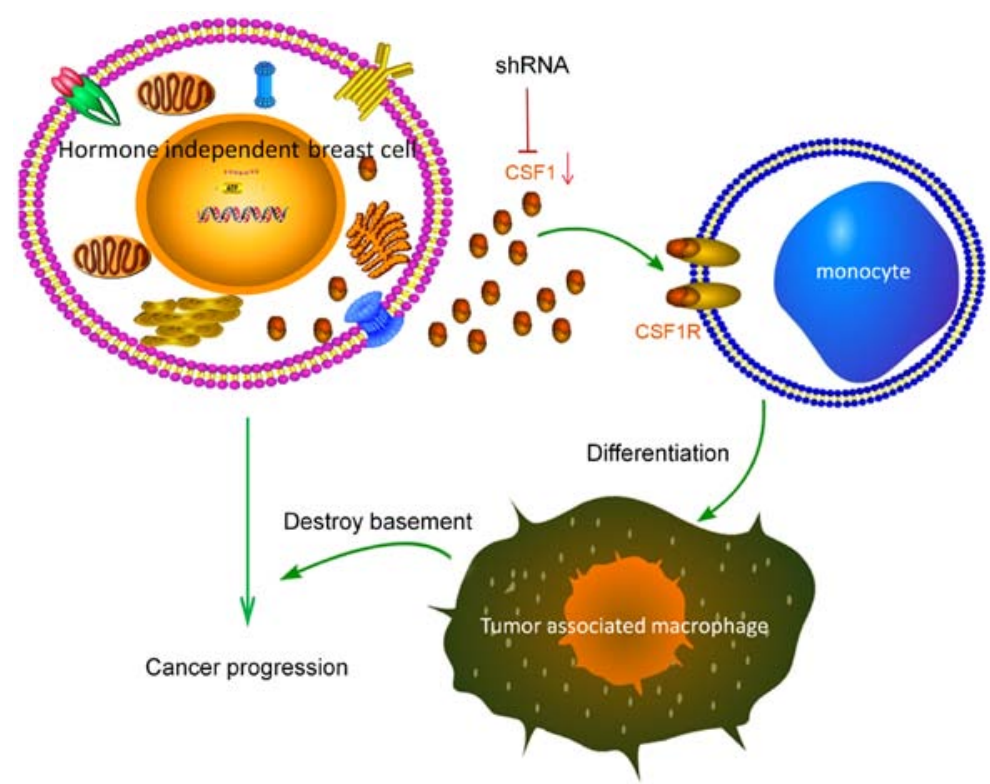

Figure 8. The schematic representation of hormone-independent breast cancer progression. Hormone-independent breast cancer cells secrete a large amount of CSF1 to build up the special tumor niche. Monocytes leave the vasculature and enter the tumor tissue in response to the cytokine secreted from the tumor, where they differentiate into TAMs. TAMs act as an important contributor to cancer progression through destroying the basement. CSF1, colony-stimulating factor 1; TAMs, tumor-associated macrophages.

that CSF1-overexpressing MCF-7 stable cells did not make U937 cells to differentiate into M2 macrophage (data not shown). This seems to be contradictory superficially, however, as mentioned previously, the active form of CSF1 is an extracellular disulfide-linked homodimer, which is evidenced by our data. No obvious increased CSF1 secretion profile in CSF1-overexpressing MCF-7 stable cell conditioned medium, since we did not envelope the CSF1 plasmid with a proper signal peptide.

Subsequently, we knocked down CSF1 in MDA-MB-231 cells using a series of siRNA sequences (Table III) specifically targeting the CSF1 gene using shRNA lentivirus particles following the protocol. The CSF1-knockdown MDA-MB-231 cells were identified by real-time PCR, which showed that CSF1 was knocked down somewhat in MDA-MB-231 artificially (Fig. 7D). We applied the most satisfactory shRNA lentivirus particles (cat. nos. ST3071-A and pLVT742) to generate a stable knockdown cell line for further study. Our data suggested that CSF1-knockdown stable MDA-MB-231 cells secrete much less CSF1 in its CM and thus failed to induce U937 cell differentiation into M2 macrophages.
In an in vivo tumorigenic ability study, $5 \times 10^{5}$ cells suspended in $100 \mu \mathrm{l}$ PBS were injected orthotopically into the mammary fat pad of 8-week-old female NOD/SCID mice. Similarly, only MDA-MB-231 cell lines form a palpable xenograft tumor successfully in 6 weeks, while being sacrificed in 8 weeks was confirmed again (Fig. 7E). Both the results of in vitro and in vivo studies confirmed that CSF1 played its role in promoting tumor progression by inducing the differentiation of monocytes.

\section{Discussion}

Many pre-clinical and clinical studies demonstrate an inverse correlation between TAM infiltration and patient prognosis indicating a macrophage-supporting role for tumor progression (23-25). The recruitment of host stromal cells, such as macrophages and mesenchymal stem cells (MSCs), to the primary tumor is a vital step towards cancer malignancy.

Breast cancer metastasis transforms a local disease which is cured by surgical excision into a systemic disease which responds poorly to available therapies and is the major 
cause of patient mortality (26). Multiple stromal cell types, including TAMs, are recruited to the TME and play their roles in metastasis $(27,28)$. TAM infiltration is quite common in breast cancer, and sometimes outnumbers the cancer cells in certain cases $(29,30)$. The abundance of TAMs in primary breast cancer biopsies is correlated with metastasis and patient mortality (30-32). In mouse models, CSF1 secreted by breast cancer cells binds to their cognate receptors, CSF1 receptor (CSF1R) and CCR2 on TAMs, leading to their recruitment to the TME, where they interact with cancer cells to promote invasion and metastasis (33-36).

In breast cancer, it is reported that the density of macrophage infiltration and abundance of genes associated with macrophage infiltration are part of a molecular signature that heralds negative prognosis in node-negative, tamoxifen-treated breast carcinomas (37). Though the expression of M2 macrophage-specific antigen CD163 varied significantly in primary breast cancer, its prevalence has a prognostic impact on OS (38).

In this study, we applied the U937 cell line as a model to investigate the correlation between monocytes and breast cancer cells. Although the use of human peripheral blood monocyte primary cells should allow stronger conclusions to be drawn, the enrichment of human peripheral blood monocytes involves many ethical issues in our institute. U937 cells have been widely used in macrophage research and have been shown to closely model primary macrophages extracted from whole blood (39-41). Moreover, we demonstrated an increased TAM infiltration within ER-negative breast cancer, and confirmed that the density of macrophage infiltration is associated with poor prognosis.

In in vitro models, using co-culture experiments, we also successfully established the differentiation and polarization of monocytes in breast cancer $\mathrm{CM}$ as confirmed by morphology and gene expression profile. Our data showed that hormone-independent breast cancer cell $\mathrm{CM}$ can induce U937 cell differentiation and then recruit it, whilst in hormone-dependent breast cancer cells no such phenomenon was observed using a panel of representing breast cancer cell lines MCF-7, MDA-MB-231 and MDA-MB-468. Despite this, in the presence of MDA-MB-231 cells, U937 cells significantly increased invasive ability compared to in the presence of MCF-7. This suggests that the specialized M2 macrophages secrete a set of specific proteins that directly destroy the basement, which contribute to cancer invasion and metastasis. We further found that there was a much higher level of CSF1 secretion in MDA-MB-231 CM than in MCF-7, which is required for monocyte differentiation and homing. Consequently, we tentatively depict the progression of hormone-independent breast cancer in Fig. 8. Identification of this specific protein will aid in developing novel drugs to treat the pro-tumorigenic microenvironment for TNBC. Using our model we have identified a potential treatment to overcome the pro-tumorigenic effects of the macrophage microenvironment.

To the best of our knowledge, this is the first report demonstrating direct experimental evidence that hormone-independent breast cancers demonstrate more aggressively than hormone-dependent breast cancers through secreting CSF1 to educate and recruit monocytes and rebuilding a pro-tumorigenic microenvironment.
In conclusion, TAMs play a critically important role in hormone-independent breast cancer progression, and circulating monocytes are inclined to being recruited and differentiating into TAMs in special tumor niche. New strategies for cancer treatment could emerge from a better understanding of the reciprocal paracrine loop between breast cancer and the microenvironment. Consequently, preventative treatment based on the suppression of monocyte differentiation and homing might be possible. These findings suggest the future possibility of using TAMs as a novel therapeutic target in patients with anti-estrogen resistance and primary TNBC, a tumor type defined by lack of estrogen receptor, progesterone receptor and ERBB2 gene amplification with no effective therapeutic measures at present, although representing $\sim 16 \%$ of all breast cancers.

\section{Acknowledgements}

We thank Dr Ping Zhang of the Fudan University Cancer Institute Research Center for help with FACS, Dr Wentao Yang of the Clinical Pathology Center for the histological evaluation, and Professor Duan Ma of the Key Laboratory of Molecular Medicine of the Ministry of Education for providing the U937 cell line. This study was supported in part by the Jiangxi Provincial Health and Family Planning Commission [2014]18/20154010, and the Organization Department of the Central Committee of the Communist Party of China 2015 'Sunshine of the West' visiting scholar program, and also the National Basic Research Program of China (2010CB834305, 2010CB834301).

\section{References}

1. Siegel RL, Miller KD and Jemal A: Cancer statistics, 2015. CA Cancer J Clin 65: 5-29, 2015.

2. Stanley ER, Berg KL, Einstein DB, Lee PS, Pixley FJ, Wang Y and Yeung YG: Biology and action of colony-stimulating factor-1. Mol Reprod Dev 46: 4-10, 1997.

3. Stanley ER, Berg KL, Einstein DB, Lee PS and Yeung YG: The biology and action of colony stimulating factor-1. Stem Cells 12 (Suppl 1): 15-24, 1994.

4. Sapi E: The role of CSF-1 in normal physiology of mammary gland and breast cancer: An update. Exp Biol Med (Maywood) 229: $1-11,2004$.

5. Chambers AF, Groom AC and MacDonald IC: Dissemination and growth of cancer cells in metastatic sites. Nat Rev Cancer 2: 563-572, 2002

6. Hughes R, Qian BZ, Rowan C, Muthana M, Keklikoglou I, Olson OC, Tazzyman S, Danson S, Addison C, Clemons M, et al: Perivascular M2 macrophages stimulate tumor relapse after chemotherapy. Cancer Res 75: 3479-3491, 2015.

7. Sugimura K, Miyata H, Tanaka K, Takahashi T, Kurokawa Y, Yamasaki M, Nakajima K, Takiguchi S, Mori M and Doki Y: High infiltration of tumor-associated macrophages is associated with a poor response to chemotherapy and poor prognosis of patients undergoing neoadjuvant chemotherapy for esophageal cancer. J Surg Oncol 111: 752-759, 2015.

8. Sasayama T, Tanaka K, Mizowaki T, Nagashima H, Nakamizo S, Tanaka H, Nishihara M, Mizukawa K, Hirose T, Itoh T, et al: Tumor-associated macrophages associate with cerebrospinal fluid interleukin-10 and survival in primary central nervous system lymphoma (PCNSL). Brain Pathol 26: 479-487, 2016.

9. Gordon S: Alternative activation of macrophages. Nat Rev Immunol 3: 23-35, 2003.

10. Svensson J, Jenmalm MC, Matussek A, Geffers R, Berg G and Ernerudh J: Macrophages at the fetal-maternal interface express markers of alternative activation and are induced by M-CSF and IL-10. J Immunol 187: 3671-3682, 2011. 
11. Wynn TA, Chawla A and Pollard JW: Macrophage biology in development, homeostasis and disease. Nature 496: 445-455, 2013.

12. Geissmann F, Manz MG, Jung S, Sieweke MH, Merad M and Ley K: Development of monocytes, macrophages, and dendritic cells. Science 327: 656-661, 2010.

13. Park KY, Li G and Platt MO: Monocyte-derived macrophage assisted breast cancer cell invasion as a personalized, predictive metric to score metastatic risk. Sci Rep 5: 13855, 2015.

14. Yu KD, Li JJ, Di GH, Wu J, Shen ZZ and Shao ZM: A straightforward but not piecewise relationship between age and lymph node status in Chinese breast cancer patients. PLoS One 5: e11035, 2010.

15. Hannan NJ, Bambang K, Kaitu'u-Lino TJ, Konje JC and Tong S: A bioplex analysis of cytokines and chemokines in first trimester maternal plasma to screen for predictors of miscarriage. PLoS One 9: e93320, 2014.

16. Lee KS, Chung JH, Lee KH, Shin MJ, Oh BH and Hong CH: Bioplex analysis of plasma cytokines in Alzheimer's disease and mild cognitive impairment. Immunol Lett 121: 105-109, 2008.

17. Kim YW, Kim SK, Kim CS, Kim IY, Cho MY and Kim NK: Association of serum and intratumoral cytokine profiles with tumor stage and neutrophil lymphocyte ratio in colorectal cancer. Anticancer Res 34: 3481-3487, 2014.

18. Shigeoka M, Urakawa N, Nakamura T, Nishio M, Watajima T, Kuroda D, Komori T, Kakeji Y, Semba S and Yokozaki H: Tumor associated macrophage expressing CD204 is associated with tumor aggressiveness of esophageal squamous cell carcinoma. Cancer Sci 104: 1112-1119, 2013.

19. Kaku Y, Imaoka H, Morimatsu Y, Komohara Y, Ohnishi K, Oda H, Takenaka S, Matsuoka M, Kawayama T, Takeya M, et al: Overexpression of CD163, CD204 and CD206 on alveolar macrophages in the lungs of patients with severe chronic obstructive pulmonary disease. PLoS One 9: e87400, 2014.

20. Györffy B, Lanczky A, Eklund AC, Denkert C, Budczies J, Li Q and Szallasi Z: An online survival analysis tool to rapidly assess the effect of 22,277 genes on breast cancer prognosis using microarray data of 1,809 patients. Breast Cancer Res Treat 123: 725-731, 2010

21. Hass R, Bartels H, Topley N, Hadam M, Köhler L, GoppeltStrübe $M$ and Resch K: TPA-induced differentiation and adhesion of U937 cells: Changes in ultrastructure, cytoskeletal organization and expression of cell surface antigens. Eur J Cell Biol 48: 282-293, 1989.

22. Fulton RJ, McDade RL, Smith PL, Kienker LJ and Kettman JR Jr: Advanced multiplexed analysis with the FlowMetrix system. Clin Chem 43: 1749-1756, 1997.

23. Schmieder A, Michel J, Schönhaar K, Goerdt S and Schledzewski K: Differentiation and gene expression profile of tumor-associated macrophages. Semin Cancer Biol 22: 289-297, 2012.

24. Obeid E, Nanda R, Fu YX and Olopade OI: The role of tumor-associated macrophages in breast cancer progression (Review). Int J Oncol 43: 5-12, 2013.

25. Kennedy BC, Showers CR, Anderson DE, Anderson L, Canoll P, Bruce JN and Anderson RC: Tumor-associated macrophages in glioma: Friend or foe? J Oncol 2013: 486912, 2013.

26. Marino N, Woditschka S, Reed LT, Nakayama J, Mayer M, Wetzel M and Steeg PS: Breast cancer metastasis: Issues for the personalization of its prevention and treatment. Am J Pathol 183: 1084-1095, 2013.
27. Quail DF and Joyce JA: Microenvironmental regulation of tumor progression and metastasis. Nat Med 19: 1423-1437, 2013.

28. Hanahan D and Coussens LM: Accessories to the crime: Functions of cells recruited to the tumor microenvironment. Cancer Cell 21: 309-322, 2012.

29. Kelly PM, Davison RS, Bliss E and McGee JO: Macrophages in human breast disease: A quantitative immunohistochemical study. Br J Cancer 57: 174-177, 1988.

30. Medrek C, Pontén F, Jirström K and Leandersson K: The presence of tumor associated macrophages in tumor stroma as a prognostic marker for breast cancer patients. BMC Cancer 12: 306, 2012.

31. Tsutsui S, Yasuda K, Suzuki K, Tahara K, Higashi H and Era S: Macrophage infiltration and its prognostic implications in breast cancer: The relationship with VEGF expression and microvessel density. Oncol Rep 14: 425-431, 2005.

32. Chaturvedi P, Gilkes DM, Takano $\mathrm{N}$ and Semenza GL: Hypoxia-inducible factor-dependent signaling between triple-negative breast cancer cells and mesenchymal stem cells promotes macrophage recruitment. Proc Natl Acad Sci USA 111: E2120-E2129, 2014.

33. Lin EY, Nguyen AV, Russell RG and Pollard JW: Colony-stimulating factor 1 promotes progression of mammary tumors to malignancy. J Exp Med 193: 727-740, 2001.

34. Wyckoff J, Wang W, Lin EY, Wang Y, Pixley F, Stanley ER, Graf T, Pollard JW, Segall J and Condeelis J: A paracrine loop between tumor cells and macrophages is required for tumor cell migration in mammary tumors. Cancer Res 64: 7022-7029, 2004.

35. Pollard JW: Tumour-educated macrophages promote tumour progression and metastasis. Nat Rev Cancer 4: 71-78, 2004.

36. Qian BZ, Li J, Zhang H, Kitamura T, Zhang J, Campion LR, Kaiser EA, Snyder LA and Pollard JW: CCL2 recruits inflammatory monocytes to facilitate breast-tumour metastasis. Nature 475: 222-225, 2011.

37. Paik S, Shak S, Tang G, Kim C, Baker J, Cronin M, Baehner FL, Walker MG, Watson D, Park T, et al: A multigene assay to predict recurrence of tamoxifen-treated, node-negative breast cancer. $\mathrm{N}$ Engl J Med 351: 2817-2826, 2004.

38. Shabo I, Stål O, Olsson H, Doré S and Svanvik J: Breast cancer expression of CD163, a macrophage scavenger receptor, is related to early distant recurrence and reduced patient survival. Int J Cancer 123: 780-786, 2008

39. Lin JA, Lin FY and Chen TL: Bone components downregulate expression of toll-like receptor 4 on the surface of human monocytic U937 cells: A cell model for postfracture immune dysfunction. Mediators Inflamm 2015: 896576, 2015.

40. Martin-Manso G, Galli S, Ridnour LA, Tsokos M, Wink DA and Roberts DD: Thrombospondin 1 promotes tumor macrophage recruitment and enhances tumor cell cytotoxicity of differentiated U937 cells. Cancer Res 68: 7090-7099, 2008.

41. Yang XV, Banerjee Y, Fernández JA, Deguchi H, Xu X, Mosnier LO, Urbanus RT, de Groot PG, White-Adams TC, McCarty OJ, et al: Activated protein C ligation of ApoER2 (LRP8) causes Dab1-dependent signaling in U937 cells. Proc Natl Acad Sci USA 106: 274-279, 2009. 